\title{
Keynote Session: Reducing Racial Disparities in Oncology-Recommendations for Implementing Guideline-Adherent Cancer Care
}

\author{
Presented by Shonta Chambers, MSW; Elizabeth Harrington; \\ Lisa A. Lacasse, MBA; Robert Winn, MD; and moderated by Alyssa A. Schatz, MSW
}

\begin{abstract}
Research shows that racial disparities exist in the delivery of guideline-adherent cancer care, and that non-White patients are less likely to receive guideline-concordant care than White patients, leading to worse health outcomes. However, these disparities are not often addressed. The Elevating Cancer Equity initiative aims to address these disparities through policy-change recommendations developed by a working group and informed by data from patients/caregivers and oncologists. The hope is that the results of these surveys and the resultant recommendations will be a step toward cancer care equity in the United States.
\end{abstract}

Multiple studies have shown that adherence to guidelines such as the NCCN Clinical Practice Guidelines in Oncology (NCCN Guidelines) improves various cancer care outcomes, including overall survival and quality-of-life measures. ${ }^{1-6}$ However, research also shows dramatic racial disparities in access to guideline-adherent cancer careparticularly between Black and White patients_-although significant cancer disparities also exist in the delivery of care to indigenous and Hispanic communities. ${ }^{7,8}$ Many oncologists, patients, and caregivers recognize these racial disparities, but the resulting gaps in care equity are not being sufficiently addressed. A keynote session at the NCCN 2021 Virtual Annual Conference discussed a joint initiative that is working to address these gaps.

The Elevating Cancer Equity (ECE) initiative is a collaborative program convened by the American Cancer Society Cancer Action Network, NCCN, and the National Minority Quality Forum. These organizations are united around similar missions to reduce cancer burden and improve the quality and accessibility of care, said Alyssa A. Schatz, MSW, Senior Director of Policy and Advocacy at NCCN and moderator of the session.

The root causes of racial disparities in oncology care are complex and include socioeconomic factors, social determinants of health, and discrimination in care delivery. The ECE initiative aims to better understand these root causes but also to move from discussion to action on these issues. They plan to do this through actionable policy and practice change recommendations, developed by a working group of national experts and informed by survey results from patients, caregivers, and oncologists.

"It's unsurprising that disparities and access to appropriate treatment have a real impact on care outcomes," said Ms. Schatz. "Black Americans have the highest death rate and the shortest survival of any racial or ethnic group in the United States, and studies have found that people of color are less likely to be prescribed appropriate pain management, which we know impacts quality of life."

\section{Racial Disparities in Cancer Care and the ECE Initiative}

According to Lisa A. Lacasse, MBA, President of the American Cancer Society Cancer Action Network, the ECE has 3 specific aims in addressing racial disparities in cancer care: to understand perceptions and experience of bias in care delivery, to develop actionable policy and practice change recommendations, and to move toward advocating for the implementation of those recommendations.

However, bringing these goals to fruition is easier said than done, she said. The driving purpose of the project-advancing and elevating equity in cancer care and outcomes-is held up by other intentional pillars, she reported. Paramount among those pillars is the inclusion of the patient and caregiver perspective.

"While there's certainly existing and established research about disparities in care across race, that research has often stopped short of examining individual 
experiences of discrimination in care," she said. "We knew there was a clear need for further research into understanding the individual experiences of patients, caregivers, and providers."

Not only do patients deserve their "voice at the table," those voices should also be prominent in determining successful recommendations to affect change in their individual experiences. She noted that the survey questions produced clear discrepancies between patient and caregiver perceptions of biases in care compared with those of oncologists. "But it's because we included the patient perspective that we're able to capture that well-rounded understanding of racial bias in cancer care," she said.

\section{Results of Patient, Caregiver, and Oncologist Surveys}

Elizabeth Harrington, Partner at Public Opinion Strategies, one of the nation's largest political and public affairs research firms, explained that her organization conducted 2 national online surveys on behalf of the coalition between December 14 and 21, 2020.

The first survey was completed by 300 patients with or survivors of cancer and 300 family caregivers (oversamples were conducted to reach 227 African Americans and 238 Hispanics across these audiences). The second survey was completed by 208 oncologists.

"The objectives of the survey were to assess perceptions about health disparities and racial bias in cancer care, exploring both the patient and caregiver perspective as well as the oncologist perspective, in terms of their experiences in providing or receiving cancer care," she said.

Respondents of the 2 surveys were asked the same 14 questions: patients and caregivers were asked how often they received these aspects of cancer care from their oncologists, and oncologists were asked how often they provided them.

Patients and caregivers were also asked whether they had experienced negative encounters with their oncologist or care team. The surveys revealed stark differences between African American and Hispanic patients compared with White patients. Nearly half of patients or caregivers reported experiencing at least one negative situations, but African American and Hispanic patients and caregivers were much more likely to have experienced negative situations with their cancer care team than were White patients.

"A significant majority of these populations [African American and Hispanics] say they've experienced at least one [of the polled] negative situations in their cancer care," Ms. Harrington reported.

\section{Working Group Overview}

According to Shonta Chambers, MSW, Executive Vice President of Health Equity and Community Engagement,
Patient Advocate Foundation, and Robert Winn, MD, Director, Virginia Commonwealth University Massey Cancer Center, who are Co-Chairs of the ECE Working Group, the purpose of the working group was to discuss the root causes of racial disparities in access to guideline-adherent cancer care and to uncover opportunities in which discriminatory behaviors and biases in care delivery could be disrupted by developing actionable policy and practice change recommendations. Ideally, when these recommendations are put into practice, they will result in meaningful changes in outcomes, said Ms. Chambers.

The working group comprised 17 national experts representing physicians, patients, caregivers, researchers, and industry. In advance of the working group meeting, members received the survey results and discussion guides to inform their work.

"The composition of the working group was intentional, because we realize that for equity in cancer care to be achieved, it will require engagement across the entire health ecosystem, and patients and caregivers should be at the core of these discussions," Ms. Chambers said. "It was critically important for us to have patients and caregivers actively engage in this process. Unfortunately, however, their voice and experiences are often omitted from these types of discussions, although they are directly impacted by the decisions that are made. We felt it vitally important to change that narrative by modeling the engagement needed moving forward."

\section{The Equity Report Card}

The working group discussed practice-change recommendations to improve equitable care delivery. "The conversation really focused heavily on what implementation would look like, and ultimately resulted in the development of what we are now calling the Equity Report Card," said Dr. Winn.

The Equity Report Card is intended to serve as a tool for providers, payers, and accreditation entities working to advance racially equitable practices in cancer care.

"Most importantly, this tool can also create greater transparency for our patients, and give us all an opportunity to highlight providers who are engaging in best practices around equity," he said.

The report card includes 17 different practices to advance equitable care delivery, and these practices are divided into the following categories: community engagement, accessibility of care and social determinants of health, addressing bias in care delivery, and quality and comprehensiveness of care.

"The group all agreed that these were meaningful practices in their ability to advance equitable care delivery," he said. "We realize that some of these items may seem difficult to operationalize, or may even be viewed as just another requirement that adds to the already large 
administrative burden or cost; I get it. But we urge you to consider previous transformations in our health system."

He mentioned the shift to measuring quality in oncology, a move that was met with a significant amount of provider discomfort. When the shift was first launched, healthcare providers cited multiple concerns about the administrative burden and cost, and even questioned the efficacy of these efforts.

"But we all know this transformation was ultimately embraced, because we knew it would be good for the people we served," he said. "We need to now approach racial disparities in the healthcare system with at least the same urgency and a commitment to systems transformation. Although it's not going to be perfect or even a complete tool at the beginning, it's our hope that this report card is at least a positive step toward that transformation."

Ms. Chambers added that the Equity Report Card does not exist in a vacuum, and that structural and systemic contributors to disparities must also be addressed. "Many of these recommendations will only be able to be widely adopted when policy catches up with practice," she said.

Recognizing this, the working group developed 16 policy change recommendations for action by Congress, payers including CMS, federal agencies and state governments, and members of the ECE initiative. These groups will work to put these recommendations into place through advocacy and outreach efforts. The full list of recommendations can be found at nccn.org/policy.

Disclosures: Ms. Harrington has disclosed having an equity interest and stock options in Pfizer Inc. Dr. Winn has disclosed receiving honoraria from Genentech, Inc. The remaining presenters and moderator have disclosed that they have not received any financial consideration from any person or organization to support this presentation.

Funding: The Elevating Cancer Equity initiative is funded by grants from AbbVie, Bluebird bio, Bristol Myers Squibb, Genentech, GlaxoSmithKline, Janssen, Lilly, Merck, MorphoSys, Pfizer, Regeneron, Sanofi, and Taiho.

Correspondence: Alyssa A. Schatz, MSW, National Comprehensive Cancer Network, 3025 Chemical Road, Suite 100, Plymouth Meeting, PA 19462 Email: Schatz@nccn.org

\section{References}

1. Foster JE, Velasco JM, Hieken TJ. Adverse outcomes associated with noncompliance with melanoma treatment guidelines. Ann Surg Oncol 2008;15:2395-2402.

2. Visser BC, Ma Y, Zak Y, et al. Failure to comply with NCCN Guidelines for the management of pancreatic cancer compromises outcomes. HPB (Oxford) 2012;14:539-547.

3. Bristow RE, Powell MA, Al-Hammadi N, et al. Disparities in ovarian cancer care quality and survival according to race and socioeconomic status. J Natl Cancer Inst 2013;105:823-832.

4. Bristow RE, Chang J, Ziogas A, et al. High-volume ovarian cancer care: survival impact and disparities in access for advanced-stage disease. Gynecol Oncol 2014;132:403-410.

5. Mearis M, Shega JW, Knoebel RW. Does adherence to National Comprehensive Cancer Network guidelines improve pain-related outcomes? An evaluation of inpatient cancer pain management at an academic medical center. J Pain Symptom Manage 2013;48: 451-458.

6. Schwam ZG, Sosa JA, Roman S, Judson BL. Receipt of care discordant with practice guidelines is associated with compromised overall survival in nasopharyngeal carcinoma. Clin Oncol (R Coll Radiol) 2016;28:402-409.

7. Lumpkins CY, Philp A, Nelson KL. A road map for the future: an exploration of attitudes, perceptions, and beliefs among African Americans to tailor health promotion of cancer-related genetic counseling and testing J Genet Couns 2020;29:518-529.

8. Meghani SH, Byun E, Gallagher RM. Time to take stock: a meta-analysis and systematic review of analgesic treatment disparities for pain in the United States. Pain Med 2012;13:150-174 